\title{
Feasibility of Outpatient Chemotherapy with S-1 and Cisplatin for Gastric Cancer
}

\author{
Hironaga Satake, Akihito Tsuji, Takeshi Kotake, Mikio Fujita \\ Department of Oncology, Kobe City Medical Center General Hospital, Kobe, Japan \\ Email: hsatake@kcho.co.jp
}

Received 6 May 2014; revised 6 June 2014; accepted 1 July 2014

Copyright (C) 2014 by authors and Scientific Research Publishing Inc.

This work is licensed under the Creative Commons Attribution International License (CC BY).

http://creativecommons.org/licenses/by/4.0/

(c) (i) Open Access

\begin{abstract}
Objective: To evaluate the feasibility of $\mathrm{S}-1$ and high-dose cisplatin short hydration regimens for outpatients with unresectable metastatic gastric cancer. Methods: Data for individual outpatients treated in our institution were retrospectively pooled to assess the feasibility of an $S-1$ and highdose cisplatin short hydration regimen (S-1: 80 to $120 \mathrm{mg}$ on Days 1 to 21; cisplatin: $60 \mathrm{mg} / \mathrm{m}^{2}$ on Day 8, every 5 weeks), which included $2250 \mathrm{ml}$ of intravenous fluids and $1000 \mathrm{ml}$ oral hydration. Ten consecutive patients were treated with $S-1$ and high-dose cisplatin short hydration for unresectable metastatic gastric cancer from July 2011 to May 2012 and were included in the analysis. Results: With a median of 3.5 medication cycles, unscheduled admission occurred in two patients for 5 days each due to paralytic ileus and cerebral infarction. Four patients required dose reduction, in both $S-1$ and cisplatin in two patients, and in S-1 alone and cisplatin alone in one patient each. Renal function transiently declined after administration of cisplatin, but serum creatinine level and estimated glomerular filtration rate were both improved by the time of the next administration. Conclusion: This study suggests that an S-1 and high-dose cisplatin short hydration strategy for outpatients with unresectable metastatic gastric cancer might be feasible.
\end{abstract}

\section{Keywords}

Gastric Cancer, Cisplatin, S-1, Short Hydration, Outpatient Chemotherapy

\section{Introduction}

Gastric cancer is one of the most common causes of death in Japan. Standard treatment for unresectable metastatic gastric cancer consists of systemic chemotherapy, and the standard regimen consists of the platinum analogue cisplatin combined with 5-fluorouracil, particularly S-1. However, the administration of cisplatin is limited by nephrotoxicity, the dose-limiting toxicity of this agent. In Japan, to reduce nephrotoxicity, a 24-h hydration 
period is recommended following administration. A number of trials have reported that hydration with $2 \mathrm{~L}$ and diuresis is sufficient to ensure the safety of cisplatin administration [1] [2], and that the addition of magnesium is effective in reducing this toxicity [3] [4]. From July 2011, we utilized an S-1 and high-dose cisplatin (SP) short hydration regimen based on the National Comprehensive Cancer Network (NCCN) Guidelines Template for patients with unresectable metastatic gastric cancer, and shifted administration from an inpatient to an outpatient basis. To our knowledge, only one report of outpatient chemotherapy with SP in patients with gastric cancer has been published in Asian, including Japan [5], however, the transition of renal function is unclear. Here, we retrospectively evaluated outpatient chemotherapy with SP short hydration for gastric cancer.

\section{Materials and Methods}

From July 2011 to May 2012, ten consecutive patients were treated with the SP short hydration regimen for metastatic gastric cancer at our institution underwritten informed consent. Data were retrospectively collected from medical charts, including past and present history; laboratory data for the evaluation of hepatic, renal, and bone marrow function; and physiological examinations. We also collected radiological examinations as follows: computed tomography (CT) of the chest, abdomen, and pelvis, and esophagogastroduodenoscopy (EGD) with biopsy to confirm histology.

All patients received oral (p.o.) administration of S-1 at a total daily dose of $80-120 \mathrm{mg}$, depending on body surface area, for 3 consecutive weeks, and a single intravenous (i.v.) administration of $60 \mathrm{mg} / \mathrm{m}^{2}$ cisplatin on Day 8. On the day of cisplatin administration, patients also received 2250 ml hydration i.v. containing $5 \mathrm{mEq}$ total magnesium and $1000 \mathrm{ml}$ hydration p.o. (Figure 1). Each cycle was followed by a 2-week rest period. Palonosetron $0.25 \mathrm{mg}$ i.v., dexamethasone $20 \mathrm{mg}$ i.v. and aprepitant $125 \mathrm{mg}$ p.o. were administered $1 \mathrm{~h}$ before chemotherapy on Day 8. Aprepitant $80 \mathrm{mg}$ p.o. and dexamethasone $4 \mathrm{mg}$ p.o. were administered on Days 2 and 3. S-1 dose was calculated according to patient body surface area as follows: less than $1.25 \mathrm{~m}^{2}, 40 \mathrm{mg} ; 1.25$ - 1.5 $\mathrm{m}^{2}, 50 \mathrm{mg}$; and greater than $1.5 \mathrm{~m}^{2}, 60 \mathrm{mg}$. Treatment was continued until one of the following occurred: progressive disease, unacceptable toxicity, patient refusal, or termination of treatment by the attending physician. Follow-up was conducted every one to four weeks. Each follow-up included a physical examination and blood study. A CT scan was conducted every four to six weeks. Adverse events were graded according to the National Cancer Institute Common Toxicity criteria (CTCAE; version 3.0). Renal function was assessed based on serum creatinine (SCr) level and estimated glomerular filtration rate (eGFR) by blood examination every week during

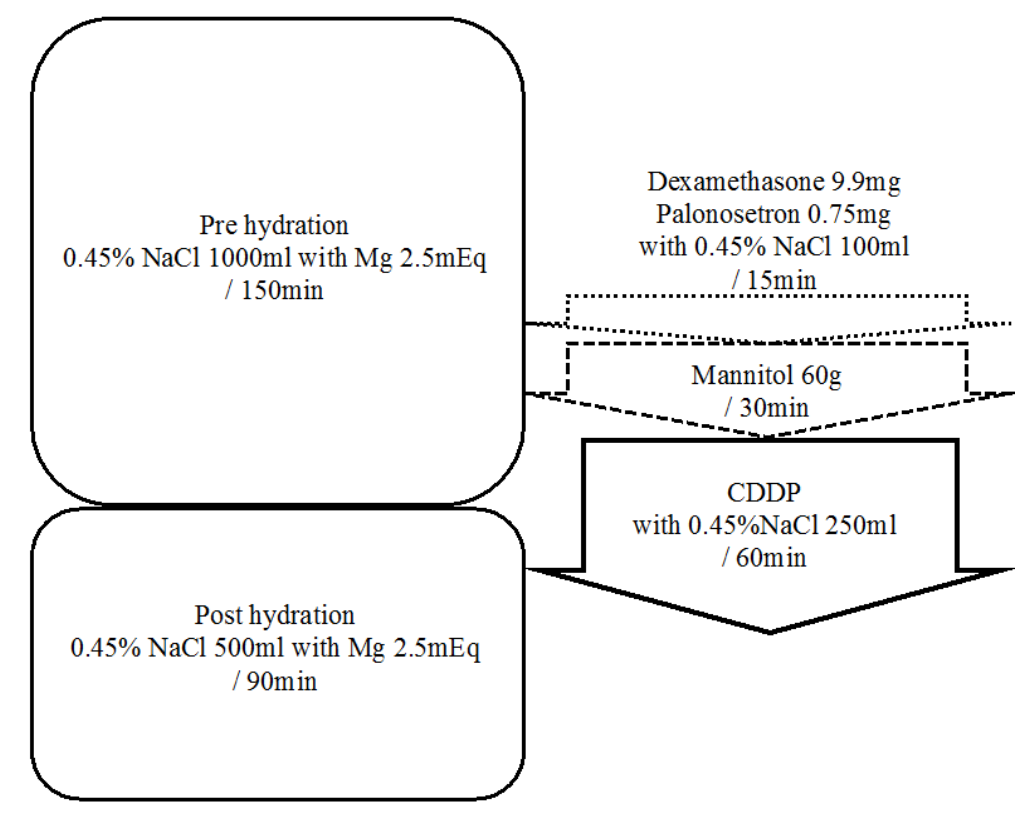

total $2250 \mathrm{ml} / 240 \mathrm{~min}$ infusion with $1000 \mathrm{ml} /$ orally hydration per day

Figure 1. Cisplatin administration. $\mathrm{NaCl}$, sodium chloride; $\mathrm{Mg}$, magnesium; min, minutes; CDDP, cisplatin. 
the $1^{\text {st }}$ cycle, and before administration of cisplatin on Day 8 in every course from the $2^{\text {nd }}$ cycle. The study was approved by the Institutional Review Board of our institution.

\section{Results}

\subsection{Baseline Characteristics}

Baseline characteristics are listed in Table 1. Disease status was unresectable in seven patients and post-operative recurrence in three. The site of metastasis was the liver in two cases and the testis in one. Two patients had received previous adjuvant chemotherapy with S-1.

\subsection{Treatment Compliance and Continuation}

Treatment compliance and response are summarized in Table 2. The median number of medication cycles was 3.5. Unscheduled admission occurred in two patients, both for 5 days, due to paralytic ileus and cerebral infarction in one patient each. Both were treated with conservative therapies. The patient with cerebral infarction had a history of previous cerebral infarction. Lacunar infarction was discovered following the onset of transient aphasia on Day 15 from the start of the $2^{\text {nd }}$ SP short hydration course, and was treated with anticoagulant agent warfarin potassium. The patient with paralytic ileus was a post-operative case who had a history of paralytic ileus

Table 1. Baseline characteristics.

\begin{tabular}{ccc}
\hline Characteristic & & $\mathrm{n}=10$ \\
Age (yr) & Median (range) & $66.5(56-76)$ \\
Gender & Male/Female & $8 / 2$ \\
ECOG PS & $0 / 1$ & $7 / 2$ \\
Surgery & Unresectable/Recurrence & $2 / 6 / 2$ \\
HER2 status & Positive/Negative/Unknown & $7 / 3$ \\
Metastatic site & $0 / 1$ & 4 \\
Peritoneal dissemination & Present & 2 \\
Pre-chemotherapy & Adjuvant S-1
\end{tabular}

ECOG PS, Eastern Cooperative Oncology Group performance status; HER2, human epidermal growth factor receptor type 2.

Table 2. Treatment compliance and response.

\begin{tabular}{cc}
\hline $\begin{array}{c}\text { (range) } \\
\text { Total chemotherapy cycle, median }\end{array}$ & $\mathrm{n}=10$ \\
Unexpected administration, n (\%) & 2 - 9) \\
Days, mean (range) & 5 days (5) \\
Reason & Paralytic ileus \\
Cerebral infarction \\
Dose reduction, n (\%) & $4(40)$ \\
S-1, CDDP & 2 (20) \\
CDDP alone & $1(10)$ \\
Extension, $\mathrm{n}(\%)$ & $1(10)$ \\
Reason & $1(10)$ \\
Interruption, $\mathrm{n}(\%)$ & Leukopenia \\
Reason & $4(40)$ \\
& PD 1 \\
AE 3
\end{tabular}

CDDP, cisplatin; PD, progressive disease; AE, adverse event. 
after a previous operation. The paralytic ileus was improved with conservative treatment.

Four patients required a dose reduction, as follows: reduction in both S-1 and cisplatin due to Grade 2 neutropenia and Grade 2 fatigue $(n=2)$; reduction in S-1 alone due to Grade 3 anemia $(n=1)$; and reduction in cisplatin alone due to Grade 2 appetite loss $(n=1)$. Treatment was extended in one patient due to Grade 3 leukopenia, and interrupted in four patients due to progressive disease $(n=1)$, and adverse events, including diarrhea ( $n$ $=1)$, cerebral infarction $(n=1)$ and peripheral sensory neuropathy $(n=1)$.

\subsection{Toxicity}

Acute toxicities are listed in Table 3 and Table 4. Acute Grade 3 or 4 hematologic toxicity included leukopenia in $10 \%(1 / 10)$, neutropenia in $10 \%(1 / 10)$ and anemia in $30 \%$. Acute Grade 3 or 4 non-hematologic toxicity included anorexia in $10 \%(1 / 10)$, paralytic ileus in $10 \%(1 / 10)$ and cerebral infarction in $10 \%(1 / 10)$. There were no cases of vomiting of any grade and no treatment-related deaths. Changes in serum creatinine (SCr) and estimated glomerular filtration rate (eGFR) are shown in Figure 2 and Table 5. Renal function transiently declined after administration of cisplatin. However, SCr and eGFR were improved by the time of the next administration.

\section{Discussion}

In this study, we observed that renal function transiently declined following the administration of cisplatin, but improved by the time of the next administration. The incidence of Grade 3 or 4 adverse events was $10 \%-30 \%$

Table 3. Hematological toxicities during chemotherapy $(n=10)$.

\begin{tabular}{|c|c|c|c|c|c|}
\hline \multirow{2}{*}{ Adverse Event } & \multicolumn{5}{|c|}{ NCI-CTC Grade } \\
\hline & 1 & 2 & 3 & 4 & $3 / 4(\%)$ \\
\hline Leukopenia & 2 & 2 & 1 & 0 & 10 \\
\hline Neutropenia & 2 & 3 & 1 & 0 & 10 \\
\hline Anemia & 1 & 2 & 3 & 0 & 30 \\
\hline Thrombocytopenia & 1 & 1 & 0 & 0 & 0 \\
\hline Creatinine & 2 & 0 & 0 & 0 & 0 \\
\hline
\end{tabular}

Table 4. Non-hematological toxicities during chemotherapy $(n=10)$.

\begin{tabular}{|c|c|c|c|c|c|}
\hline \multirow{2}{*}{ Adverse event } & \multicolumn{5}{|c|}{ NCI-CTC Grade } \\
\hline & 1 & 2 & 3 & 4 & $3 / 4(\%)$ \\
\hline Anorexia & 3 & 3 & 1 & 0 & 10 \\
\hline Cerebral infarction & 0 & 0 & 1 & 0 & 10 \\
\hline Constipation & 2 & 1 & 0 & 0 & 0 \\
\hline Diarrhea & 1 & 3 & 0 & 0 & 0 \\
\hline Dysgeusia & 1 & 0 & 0 & 0 & 0 \\
\hline Fatigue & 4 & 3 & 0 & 0 & 0 \\
\hline Fever & 1 & 0 & 0 & 0 & 0 \\
\hline Hiccups & 1 & 0 & 0 & 0 & 0 \\
\hline Mucositis oral & 0 & 2 & 0 & 0 & 0 \\
\hline Nausea & 2 & 2 & 0 & 0 & 0 \\
\hline Paralytic ileus & 0 & 0 & 1 & 0 & 10 \\
\hline Peripheral sensory neuropathy & 0 & 1 & 0 & 0 & 0 \\
\hline Pruritus & 1 & 0 & 0 & 0 & 0 \\
\hline Rash & 1 & 0 & 0 & 0 & 0 \\
\hline Vomiting & 0 & 0 & 0 & 0 & 0 \\
\hline
\end{tabular}


Table 5. Transition of serum creatinine (SCr) and estimated glomerular filtration rate (eGFR).

\begin{tabular}{ccc}
\hline & SCr $(\mathrm{mg} / \mathrm{dl})$ & eGFR $(\mathrm{mL} / \mathrm{min})$ \\
\cline { 2 - 3 } & Mean \pm SE & Mean \pm SE \\
\hline Cycle 1 Day 8 & $0.69 \pm 0.15$ & $87.8 \pm 12.4$ \\
Cycle 1 Day 15 & $0.93 \pm 0.24$ & $72.5 \pm 15.6$ \\
Cycle 1 Day 22 & $0.74 \pm 0.12$ & $76.2 \pm 16.0$ \\
Cycle 2 Day 8 & $0.82 \pm 0.22$ & $74.6 \pm 16.6$ \\
Cycle 3 Day 8 & $0.84 \pm 0.24$ & $76.3 \pm 15.7$ \\
Cycle 4 Day 8 & $0.79 \pm 0.27$ & $83.8 \pm 15.8$ \\
Cycle 5 Day 8 & $0.85 \pm 0.24$ & $67.3 \pm 13.5$ \\
Cycle 6 Day 8 & $0.83 \pm 0.23$ & $69.5 \pm 16.3$ \\
Cycle 7 Day 8 & $0.92 \pm 0.32$ & $68.0 \pm 19.1$ \\
Cycle 8 Day 8 & $0.96 \pm 0.33$ & $66.5 \pm 23.3$ \\
\hline
\end{tabular}

SCr, serum creatinine; eGFR, estimated glomerular filtration rate; SE, standard error.

(a)

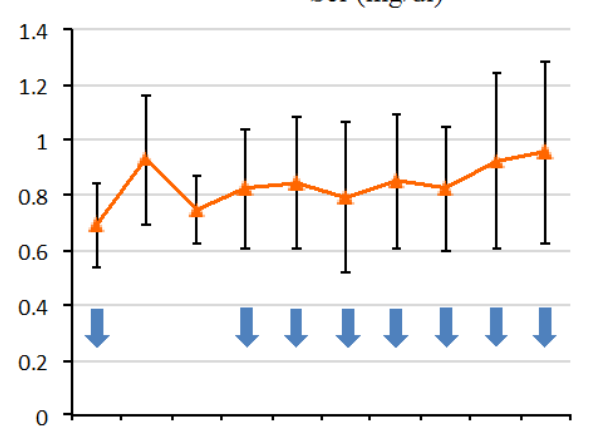

(b)

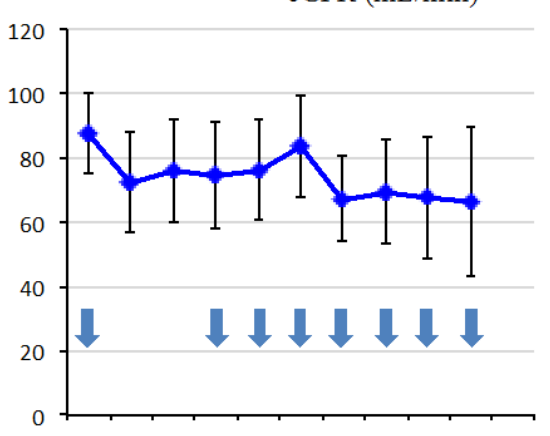

\section{$\downarrow$ CDDPadministration}

Figure 2. Changes in of serum creatinine level and estimated glomerular filtration rate ( $\mathrm{n}=$ 10). (a) Change in serum creatinine; (b) Change in estimated glomerular filtration rate.

for myelosuppression, $10 \%$ for anorexia, $10 \%$ for paralytic ileus and $10 \%$ for cerebral infarction. In the SPIRITS trial, a cornerstone Phase III trial of S-1 plus cisplatin for metastatic gastric cancer, Grade 3 or 4 adverse events in the SP arm were as follows: leucopenia (11\%), neutropenia (40\%), anemia (26\%), thrombocytopenia (5\%), febrile neutropenia (3\%), anorexia (30\%), nausea (11\%), fatigue (4\%), vomiting (4\%), diarrhea (4\%), stomatitis $(0.7 \%)$, rash (2\%) and hyponatremia (3\%) [6]. Allowing for the substantial limitations of our study, these results are considered comparable with the previous study.

The optimal intravenous solution and regimen for hydration are unclear, but in general at least $2 \mathrm{~L}$ of i.v. saline is required on the day of cisplatin administration to maintain good urine flow. This was therefore only conducted on the day of cisplatin administration. In Italy, Tiseo et al. (2007) also reported that a short period of hydration, including $2000 \mathrm{ml}$ of fluid with control of diuresis, is feasible [7]. One reason for hydration is the critical importance of maintaining urine flow within $4-6 \mathrm{~h}$ of cisplatin administration to prevent nephrotoxicity. Approximately $90 \%$ of platinum becomes irreversibly bound to plasma protein within $4 \mathrm{~h}$ after cisplatin administration, and the plasma concentration of free platinum, which induces nephrotoxicity, decreases almost to the limit of quantification within 4 - $6 \mathrm{~h}$ after administration [8]. Most countries have accordingly started to administer cisplatin on an outpatient basis, but this change has yet to occur in Japan [9]. The development of the antiemetic drug aprepitant, a potent and selective antagonist of the neurokinin (NK)1 neurotransmitter receptor, and palonosetron, a second generation 5-hydroxytryptamine 3 (5-HT(3))-receptor antagonist, prevent chemotherapy- 
induced nausea and vomiting (CINV)] [10]-[12]. In our study, we administered aprepitant, palonosetron and dexamethasone to prevent CINV, and Grade 3 or 4 nausea was not observed. This in turn allowed the oral intake of food and water, and acceptable nephrotoxicity.

In economic terms, inpatient chemotherapy with a high-dose cisplatin regimen usually requires at least three days of administration, giving a medical cost in Japan of at least US \$1200 for every cycle. In contrast, outpatient chemotherapy with high-dose cisplatin in combination with aprepitant plus palonosetron and dexamethasone as antiemetic prophylaxis costs approximately $\$ 700$ per cycle in Japan. Total administration time of each SP short hydration regimen, which included $2250 \mathrm{ml}$ of fluids with diuresis, was approximately 4 hours, allowing the management of the same regimen twice per day in the outpatient department.

Limitations of the study include its small number of patients and single institution, retrospective study design.

This study suggests that the SP short hydration strategy might be feasible in Japanese patients with gastric cancer, and can provide time saving for patients and reduce medical costs. A Phase II study of S-1 and high-dose cisplatin chemotherapy with short hydration for unresectable metastatic gastric cancer is now ongoing as part of the Japan Southwest Research Support Organization Group trial (JWOG-G1 trial).

\section{References}

[1] Stewart, D.J., Dulberg, C.S., Mikhael, N.Z., Redmond, M.D., Montpetit, V.A. and Goel, R. (1997) Association of Cisplatin Nephrotoxicity with Patient Characteristics and Cisplatin Administration Methods. Cancer Chemotherapy and Pharmacology, 40, 293-308. http://dx.doi.org/10.1007/s002800050661

[2] Vogl, S.E., Zaravinos, T. and Kaplan, B.H. (1980) Toxicity of Cis-Diamminedichloroplatinum II Given in a Two-Hour Outpatient Regimen of Diuresis and Hydration. Cancer, 45, 11-15.

http://dx.doi.org/10.1002/1097-0142(19800101)45:1<11::AID-CNCR2820450104>3.0.CO;2-E

[3] Willox, J.C., McAllister, E.J., Sangster, G. and Kaye, S.B. (1986) Effects of Magnesium Supplementation in Testicular Cancer Patients Receiving Cis-Platin: A Randomised Trial. British Journal of Cancer, 54, 19-23. http://dx.doi.org/10.1038/bjc.1986.147

[4] Bodnar, L., Wcislo, G., Gasowska-Bodnar, A., Synowiec, A., Szarlej-Wcislo, K. and Szczylik, C. (2008) Renal Protection with Magnesium Subcarbonate and Magnesium Sulphate in Patients with Epithelial Ovarian Cancer after Cisplatin and Paclitaxel Chemotherapy: A Randomised Phase II Study. European Journal of Cancer, 44, 2608-2614. http://dx.doi.org/10.1016/j.ejca.2008.08.005

[5] Okazaki, S., Nakajima, T.E., Hashimoto, J., Yamamoto, S., Takahari, D., Kato, K., Hamaguchi, T., Yamada, Y., Shimada, Y. and Tamura, K. (2013) A Feasibility Study of Outpatient Chemotherapy with s-1 + Cisplatin in Patients with Advanced Gastric Cancer. Gastric Cancer, 16, 41-47. http://dx.doi.org/10.1007/s10120-012-0139-4

[6] Koizumi, W., Narahara, H., Hara, T., Takagane, A., Akiya, T., Takagi, M., Miyashita, K., Nishizaki, T., Kobayashi, O., Takiyama, W., Toh, Y., Nagaie, T., Takagi, S., Yamamura, Y., Yanaoka, K., Orita, H. and Takeuchi, M. (2008) S-1 plus Cisplatin versus s-1 Alone for First-Line Treatment of Advanced Gastric Cancer (Spirits Trial): A Phase III Trial. The Lancet Oncology, 9, 215-221. http://dx.doi.org/10.1016/S1470-2045(08)70035-4

[7] Tiseo, M., Martelli, O., Mancuso, A., Sormani, M.P., Bruzzi, P., Di Salvia, R., De Marinis, F. and Ardizzoni, A. (2007) Short Hydration Regimen and Nephrotoxicity of Intermediate to High-Dose Cisplatin-Based Chemotherapy for Outpatient Treatment in Lung Cancer and Mesothelioma. Tumori, 93, 138-144.

[8] Sasaki, Y., Tamura, T., Eguchi, K., Shinkai, T., Fujiwara, Y., Fukuda, M., Ohe, Y., Bungo, M., Horichi, N., Niimi, S., Minato, K. and Nakagawa, K. (1989) Pharmacokinetics of (Glycolate-0,0')-Diammine Platinum (II), a New Platinum Derivative, in Comparison with Cisplatin and Carboplatin. Cancer Chemotherapy and Pharmacology, 23, 243-246. http://dx.doi.org/10.1007/BF00451649

[9] Yamada, K., Yoshida, T., Zaizen, Y., Okayama, Y., Naito, Y., Yamashita, F., Takeoka, H., Mizoguchi, Y. and Azuma, K. (2011) Clinical Practice in Management of Hydration for Lung Cancer Patients Receiving Cisplatin-Based Chemotherapy in Japan: A Questionnaire Survey. Japanese Journal of Clinical Oncology, 41, 1308-1311. http://dx.doi.org/10.1093/jjco/hyr145

[10] Longo, F., Mansueto, G., Lapadula, V., Stumbo, L., Del Bene, G., Adua, D., De Filippis, L., Bonizzoni, E. and Quadrini, S. (2012) Combination of Aprepitant, Palonosetron and Dexamethasone as Antiemetic Prophylaxis in Lung Cancer Patients Receiving Multiple Cycles of Cisplatin-Based Chemotherapy. International Journal of Clinical Practice, 66, 753-757. http://dx.doi.org/10.1111/j.1742-1241.2012.02969.x

[11] Rapoport, B.L., Jordan, K., Boice, J.A., Taylor, A., Brown, C., Hardwick, J.S., Carides, A., Webb, T. and Schmoll, H.J. (2010) Aprepitant for the Prevention of Chemotherapy-Induced Nausea and Vomiting Associated with a Broad Range of Moderately Emetogenic Chemotherapies and Tumor Types: A Randomized, Double-Blind Study. Support Care Cancer, 18, 423-431. http://dx.doi.org/10.1007/s00520-009-0680-9 
[12] Saito, M., Aogi, K., Sekine, I., Yoshizawa, H., Yanagita, Y., Sakai, H., Inoue, K., Kitagawa, C., Ogura, T. and Mitsuhashi, S. (2009) Palonosetron plus Dexamethasone versus Granisetron plus Dexamethasone for Prevention of Nausea and Vomiting during Chemotherapy: A Double-Blind, Double-Dummy, Randomised, Comparative Phase III Trial. The Lancet Oncology, 10, 115-124. http://dx.doi.org/10.1016/S1470-2045(08)70313-9 
Scientific Research Publishing (SCIRP) is one of the largest Open Access journal publishers. It is currently publishing more than 200 open access, online, peer-reviewed journals covering a wide range of academic disciplines. SCIRP serves the worldwide academic communities and contributes to the progress and application of science with its publication.

Other selected journals from SCIRP are listed as below. Submit your manuscript to us via either submit@scirp.org or Online Submission Portal.
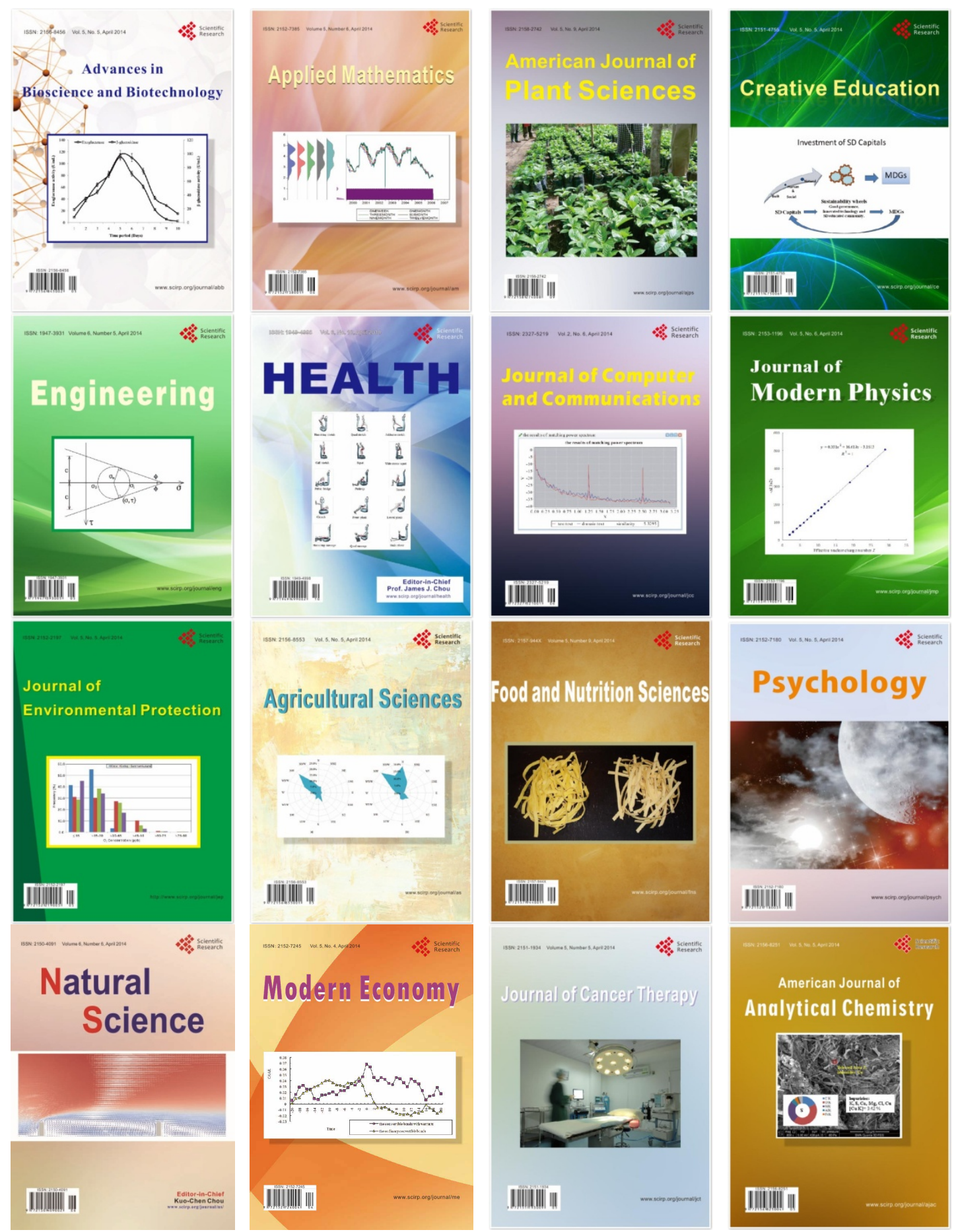